\title{
Investigation of linezolid resistance among methicillin resistant Staphylococcus aureus strains isolated from state hospitals in the East and West coast of Malaysia
}

\author{
Thiruchelvi Pulingam, Pazilah Ibrahim and Seok-Ming Toh*
}

\author{
Discipline of Pharmaceutical Technology, School of Pharmaceutical Sciences, Universiti Sains Malaysia, 11800 USM \\ Penang, Malaysia. \\ Email: smtoh@usm.my
}

Received 13 September 2013; Received in revised form16 December 2013; Accepted 20 December 2013

\begin{abstract}
Aims: Linezolid, the first available agent of a new antimicrobial class, the oxazolidinones, is broadly effective against drug-resistant gram-positive pathogens which are common causes of infections in hospitalized patients. However, it is not widely available in Malaysian government hospitals and therefore, relatively little is known about the prevalence of linezolid resistance in this background. The main aim of our study was to determine the prevalence of linezolid resistance among a total of 100 strains of methicillin-resistant Staphylococcus aureus (MRSA) isolated in June-October 2012 from two Malaysian state hospitals, namely Hospital Universiti Sains Malaysia (HUSM) and Hospital Pulau Pinang (HPP). The antibiotic susceptibility of the strains was also determined.

Methodology and results: The clinical MRSA isolates were tested by the antibiotic strip method for in vitro susceptibility to 6 antibiotics (linezolid, vancomycin, chloramphenicol, clindamycin, erythromycin and oxacillin). Using this method, we report a 2- to 3-fold greater potency of linezolid versus vancomycin, the current treatment of choice for MRSA in these institutions. Three of the isolates, all originating from HPP, showed intermediate resistance to vancomycin $(3-8 \mu \mathrm{g} / \mathrm{mL})$. Four percent of all isolates were resistant to chloramphenicol, $20 \%$ were resistant to clindamycin, 33\% were resistant to erythromycin and all were resistant to oxacillin. Multiple drug resistance was found in 21 HPP isolates with 3 different non-susceptibility profiles observed.

Conclusion, significance and impact study: Based on our data, we would recommend linezolid as an acceptable alternative to vancomycin. Linezolid therapy would be valuable in cases of treatment failure with empirical vancomycin, serious invasive infections caused by vancomycin intermediate $S$. aureus (VISA) or whenever aggressive vancomycin dosing is not recommended due to toxicity concerns.
\end{abstract}

Keywords: Antimicrobial resistance, linezolid, Malaysia, MRSA, Staphylococcus aureus

\section{INTRODUCTION}

MRSA is a worrisome pathogen in nosocomial infections in developing countries including Malaysia. Hospital Kuala Lumpur (HKL), which is the largest government tertiary referral hospital in the country, records an annual average of $40 \%$ MRSA prevalence (Ghaznavi-Rad et al., 2010). The empiric treatment of choice for MRSA infections, socalled 'conventional' treatment, has been parenteral vancomycin. Although most MRSA strains are susceptible to vancomycin, a patient who is administered the drug requires serum trough (pre-dose) vancomycin concentration to be monitored every $12 \mathrm{~h}$ due to vancomycin's narrow therapeutic range. Vancomycin trough monitoring has been recommended as the most accurate and practical method to monitor this antibiotic and as a surrogate marker for the area under curve (AUC) pharmacokinetic parameter. A trough concentration range of $15-20 \mu \mathrm{g} / \mathrm{mL}$ is targeted as the lowest level of vancomycin present in the body (Rybak et al., 2009). In patients who have renal impairment or have fluctuating volumes of distribution, such narrow concentrations are often difficult to attain. Furthermore, nephrotoxicity has been associated with high doses of vancomycin (Lodise et al., 2008). The emergence of vancomycin intermediate $S$. aureus (VISA) and vancomycin-resistant $S$. aureus (VRSA), though relatively uncommon, poses an additional challenge to the use of vancomycin (Ahmad et al., 2010). Genome sequence comparison of all available VRSA Clonal Cluster 5 (CC5) strains isolated from the 12 documented cases of VRSA in the United States of America since 2002 show that these strains possess a constellation of traits that position them for acquiring resistance to antibiotics in mixed infections (Kos et al., 2012). Hence the emergence of such superstrains with enhanced virulence poses a severe threat to our limited antibiotic arsenal and may lead to poor clinical outcomes.

The Infectious Diseases Society of America recommends that an alternative to vancomycin be used for MRSA isolates with a vancomycin MIC $>2 \mu \mathrm{g} / \mathrm{mL}$ (Liu 
et al., 2011). In this regard, members of a new class of synthetic antibiotics which targets the ribosome, called the oxazolidinones, have been suggested as a valuable alternative to vancomycin treatment. Linezolid, the first oxazolidinone to be introduced into clinical practice in the early 2000's, is used for treatment of infections caused by Gram-positive pathogens, including MRSA and VRSA. Additional advantages of linezolid over vancomycin therapy include excellent tissue penetration and equivalent bioavailability between oral and intravenous formulations.

Although MRSA is a frequently isolated pathogen in Malaysian hospitals, there is very limited data regarding the use of alternative therapies to empiric vancomycin treatment. Alternative therapies such as linezolid are not readily available in Malaysian government hospitals because vancomycin is still largely effective against MRSA while in terms of acquisition, the only available version of linezolid, the innovator's Zyvox ${ }^{\circledR}$ (Pfizer) is expensive. The use of linezolid in Malaysian government hospitals is restricted to select cases where excellent tissue penetration is required such as difficult-to-treat endocarditis, central nervous system infections and bone and joint infections caused by Gram-positive cocci. As such, relatively little is known about the prevalence of linezolid resistance within the Malaysian setting and the mechanism(s) through which linezolid resistance is acquired. The significant scarcity of Malaysian studies conducted to date on linezolid for MRSA treatment is compounded by variability in the methods used between antibiotic surveillance studies, thus limiting comparison between hospitals.

The aim of our study was to investigate the prevalence of linezolid resistance among MRSA isolates from the largest state tertiary referral hospitals in two geographic areas: one being the West coast state of Penang (Hospital Pulau Pinang [HPP]) and the other, the East coast state of Kelantan (Hospital Universiti Sains Malaysia [HUSM]). We also explored the multidrugresistance of these Malaysian nosocomial isolates. By gathering in vitro evidence which may support the use of alternative therapies, specifically linezolid, within the Malaysian hospital setting, we hoped to provide information for the development of guidelines for the rational use of medicines.

\section{MATERIALS AND METHODS}

Malaysia is a Southeast Asian country where the overall prevalence of MRSA in 2012 amongst 37 hospitals was $17.3 \%$, based on data in the National Surveillance of Antibiotic Resistance Report, Ministry of Health, Malaysia (available online

http://www.imr.gov.my/component/content/article/75-

national-collabration/1294-nsar.html). Our study sample comprised of 100 MRSA clinical isolates from two distinct geographical locations in Malaysia. The demographic and culture basis for MRSA infection was not determined. Sample size was based on expedience. Eighteen convenience samples were obtained from HUSM, Kubang
Kerian, Kelantan on the East coast of Peninsular Malaysia. HUSM is located in the Northeast of Peninsular Malaysia and serves as the main referral centre for the East coast of Peninsular Malaysia. Also a teaching hospital belonging to Universiti Sains Malaysia, HUSM has 747 beds, several specialist clinics, operation theatres and patient wards. The remaining 82 clinical MRSA isolates were from the West coast of Peninsular Malaysia and were collected from the Department of Pathology, HPP from June-October 2012. Hospital Pulau Pinang, the second largest government hospital in the country, is a 1107-bed referral hospital for the Northern region of the West coast of Peninsular Malaysia and has 31 specialist clinics, 17 operation theatres, and 36 patient wards. The strain collection in HPP was ended when the 4-month collection study period approved by the ethics committee expired. There is no national guideline for the rational treatment of MRSA in Malaysia. However, the mainstay of parenteral therapy for MRSA infections in the study hospitals has been vancomycin. As per Infectious Diseases Society of America (IDSA) guidelines, alternative anti-MRSA agents used in these hospital's antibiotic panel have included clindamycin, daptomycin, linezolid, quinupristin-dalfopristin, rifampin, tetracycline and co-trimoxazole (Liu et al., 2011).

S. aureus ATCC 33591 and ATCC 25923 were used as the MRSA and MSSA control strains respectively. To determine antimicrobial susceptibility, the Epsilometer test (Etest) was performed using Liofilchem ${ }^{\circledR}$ (Italy) antibiotic strips. Breakpoints for defining susceptibility categories for linezolid as well as 5 other drugs, selected on the basis of representation of different antibiotic classes and their antimicrobial activity against $S$. aureus, namely, chloramphenicol, clindamycin, erythromycin, oxacillin and vancomycin, were interpreted according to the criteria of the Clinical and Laboratory Standards Institute (CLSI, 2013). Vancomycin was included in the antimicrobial susceptibility testing since its MIC determination is not routinely carried out on clinical isolates of MRSA unless requested by the clinicians when their patients do not respond to treatment. Hence, we also wanted to determine the distribution of vancomycin MIC values among the MRSA isolates in our study hospitals and to identify putative VRSA and/or VISA strains. The study was approved by the National Institutes of Health for conducting research in the Ministry of Health Malaysia (NMRR-11-1093-8876).

\section{RESULTS AND DISCUSSION}

Figure 1 provides the average MICs for 18 MRSA isolates from HUSM and 82 MRSA isolates from HPP. All 100 strains were sensitive to linezolid with an average MIC of $0.71 \mu \mathrm{g} / \mathrm{mL}$. The MICs for the current mainstay treatment for MRSA infections in these institutions, vancomycin, averaged at $1.13 \mu \mathrm{g} / \mathrm{mL}$. Overall, a 2- to 3 -fold greater potency of linezolid versus vancomycin was observed although vancomycin still retained activity against the MRSA isolates from both hospitals. While acknowledging that the number of isolates tested from the two hospitals 
was not equal as a limitation of expedience sampling, we noted that the HPP isolates had average MICs which were slightly raised for all the antibiotics tested except for oxacillin. The HPP MRSA isolates had developed very high MICs against clindamycin (a lincosamide) and erythromycin (a macrolide) in particular. The likelihood of such cross-resistance due to $\mathrm{MLS}_{\mathrm{B}}$ phenotype (resulting from dimethylation of A2058 in 23S rRNA leading to

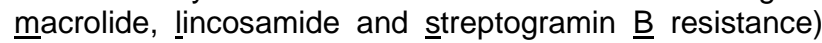
will need to be verified using erm PCR and D-test. The MICs for chloramphenicol (a phenicol) averaged at 5.06 $\mu \mathrm{g} / \mathrm{mL}$ for HUSM isolates versus $16.1 \mu \mathrm{g} / \mathrm{mL}$ for HPP isolates, making this antibiotic moderately active for HUSM isolates and only intermediately effective against MRSA from HPP.

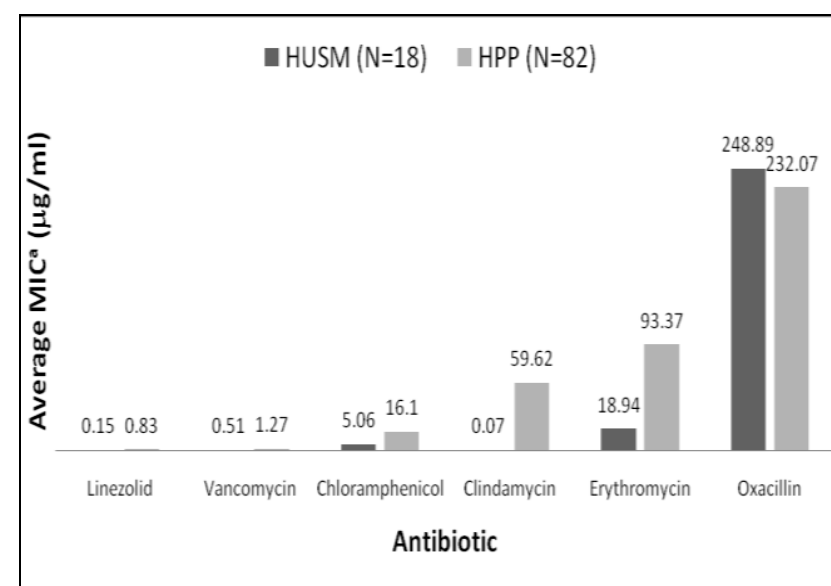

Figure 1: In vitro activity of linezolid and 5 other antibiotics against Malaysian methicillin-resistant Staphylococcus aureus (MRSA) strains from Hospital Universiti Sains Malaysia (HUSM) and Hospital Pulau Pinang (HPP). ${ }^{a} \mathrm{MICs}$ of antibiotics were determined by the CLSI method where sensitive (S), intermediate (I) and resistant $(R)$ breakpoints in $\mu \mathrm{g} / \mathrm{mL}$ is as follows: linezolid $(\mathrm{S} \leq 4 ; 4<\mathrm{l}>7 ; \mathrm{R} \geq 8)$, vancomycin $(\mathrm{S} \leq 2 ; 2<\mathrm{l}>16 ; \mathrm{R} \geq 16)$, chloramphenicol $(\mathrm{S} \leq 8 ; \quad 8<\mathrm{l}>32 ; \quad \mathrm{R} \geq 32)$, clindamycin ( $\leq \leq 0.5 ; 0.5<1>4 ; R \geq 4)$, erythromycin $(S \leq 0.5 ; 0.5<1>8 ; R \geq 8)$ and oxacillin $(S \leq 2 ; 2<1>4 ; R \geq 4)$.

Figure 2 details the distribution of the obtained MICs into sensitive, intermediate and resistant for linezolid and the 5 other antibiotics. In terms of overall resistance, as expected, all isolates were resistant to oxacillin. Thirtythree percent of the isolates were resistant to erythromycin while $20 \%$ were resistant to clindamycin. On the other hand, only $4 \%$ of all isolates were resistant to chloramphenicol. Three of the isolates, all originating from HPP, showed intermediate resistance to vancomycin with MICs ranging from $3-8 \mu \mathrm{g} / \mathrm{mL}$. All of the 100 isolates were fully susceptible to linezolid. The in vitro linezolid susceptibility of the MRSA isolates suggests the absence of linezolid resistance mechanisms. Further testing of this hypothesis is currently under investigation.

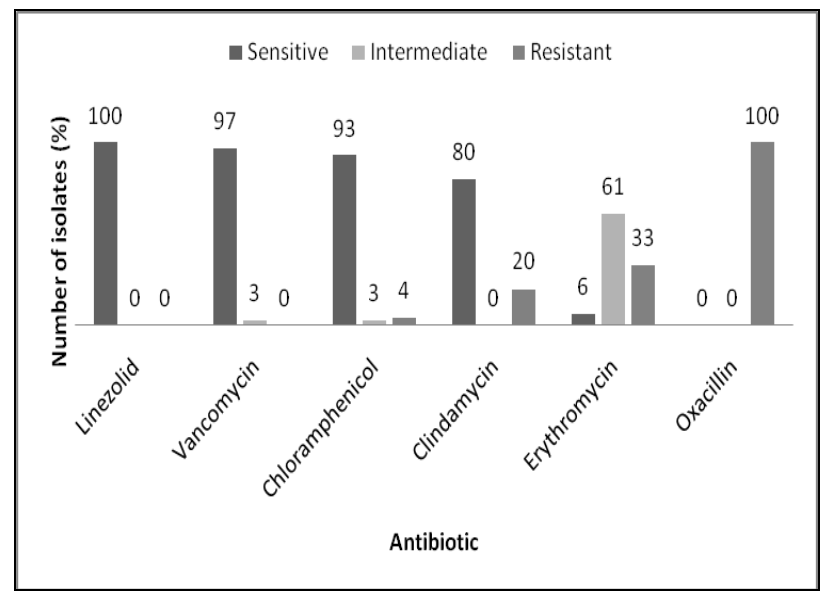

Figure 2: Distribution of MICs for linezolid and 5 other antibiotics into sensitive, intermediate and resistant categories for the 100 Malaysian methicillin-resistant Staphylococcus aureus (MRSA) isolates, as defined by CLSI interpretive criteria.

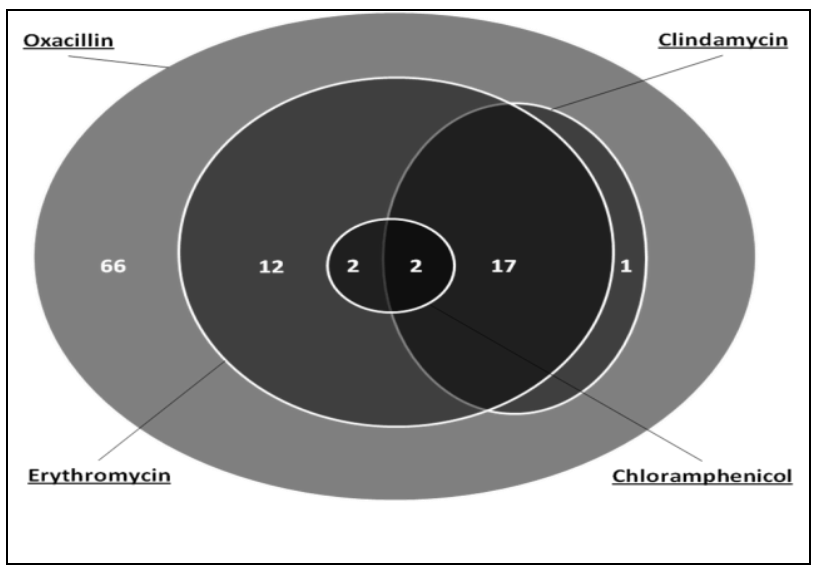

Figure 3: Subdivision of the 100 methicillin-resistant Staphylococcus aureus (MRSA) strains into 3 different multi-drug resistant (MDR) types with respect to crossresistance between four antibiotics from different classes.

Although all 100 MRSA isolates appear to be susceptible to linezolid by standard susceptibility test methods, clinical linezolid treatment failure may still be possible especially in a heterogeneously resistant isolate (Ikeda-Dantsuji et al., 2011). A recent study on 50 linezolid-resistant staphylococcal isolates from Italy showed cross-resistance to erythromycin, lincomycin, levofloxacin, gentamicin, cotrimoxazole and teicoplanin (Campanile et al., 2013). Combined resistance to phenicols, macrolides and lincosamides may have a synergistic effect on linezolid resistance since all these drugs have overlapping drug binding sites on the large ribosomal subunit (Schlunzen et al., 2001; Leach et al., 2007). 
Table 1: Prevalence of multidrug-resistance (resistance to at least one agent in three or more antimicrobial categories) in the methicillin-resistant Staphylococcus aureus (MRSA) isolates according to hospital.

\begin{tabular}{|c|c|c|c|}
\hline \multirow[b]{2}{*}{ Resistance profile } & \multicolumn{2}{|c|}{ Number of isolates from each hospital } & \multirow[b]{2}{*}{ Total (\%) } \\
\hline & $\begin{array}{c}\text { Hospital Universiti Sains } \\
\text { Malaysia (HUSM), Kelantan } \\
\mathrm{N}=18\end{array}$ & $\begin{array}{l}\text { Hospital Pulau Pinang } \\
\text { (HPP), Penang } \\
\mathrm{N}=82 \\
\end{array}$ & \\
\hline Resistant to 1 antibiotic & 17 & 49 & 66 \\
\hline Resistant to 2 antibiotics & 1 & 12 & 13 \\
\hline Resistant to 3 antibiotics & 0 & 19 & 19 \\
\hline Resistant to 4 antibiotics & 0 & 2 & 2 \\
\hline Resistant to 5 antibiotics & 0 & 0 & 0 \\
\hline Resistant to 6 antibiotics & 0 & 0 & 0 \\
\hline $\begin{array}{l}\text { Multidrug-resistance from each } \\
\text { hospital (\%) }\end{array}$ & $0(0)$ & $21(25.6)$ & $21(21.0)$ \\
\hline
\end{tabular}

Our MRSA isolates did indeed show a multidrugresistant MDR profile [defined as acquired nonsusceptibility to at least one agent in three or more antimicrobial categories (Magiorakos et al., 2012). In fact, $25.6 \%$ of the HPP isolates $(21 / 82)$ were multiple antibiotic resistant while only one of the oxacillin-resistant HUSM isolates was additionally resistant to erythromycin, making all 18 HUSM isolates non-MDR (Table 1). Among the 82 HPP MRSA strains, 3 different MDR types were found, namely, oxacillin ${ }^{R} /$ erythromycin ${ }^{R} /$ clindamycin $^{R} \quad(n=17$ strains), oxacillin ${ }^{R} /$ erythromycin ${ }^{R} /$ chloramphenicol $^{R} \quad(n=2$ strains) and oxacillin ${ }^{\mathrm{R}} /$ erythromycin $\mathrm{R} /$ chloramphenicol $^{\mathrm{R}} /$ clindamycin $^{R}$ ( $n=2$ strains) (Figure 3 ). The elucidation of the underlying resistance genes or mechanisms of multidrug-resistance is currently under investigation. The highly probable co-existence of such resistance determinants in our MDR strains of MRSA imply that linezolid-resistant strains may be very easily co-selected, especially under antibiotic pressure.

\section{CONCLUSION}

Even though the best alternative antibiotic choice is not a resolved issue, insight into the prevalence of resistance is invaluable for strategic MRSA pathogenic control. In all 100 clinical isolates from 2 Malaysian hospital sites, complete susceptibility to linezolid was found, and the results of the study provide the basis for the use of oxazolidinone therapies to address possible emerging vancomycin resistance among Malaysian hospitalacquired MRSA strains. The presence of cross-resistance to four classes of antibiotics in the MRSA isolates multiplies the risk of co-selection of linezolid-resistant strains. Additional surveillance studies are clearly warranted in order to monitor the emergence of linezolid resistance in Malaysia.

\section{ACKNOWLEDGEMENTS}

We thank Dr. Jamilah Baharom from the Department of Pathology, HPP and Dr. Siti Suraiya Md. Noor from the Department of Microbiology and Parasitology, School of Medical Sciences, Universiti Sains Malaysia for providing the MRSA isolates and Dr. Balamurugan Tangiisuran for critical reading of the manuscript. This work was supported by Research University (RU) Grant 1001/PFARMASI/815074 from Universiti Sains Malaysia.

\section{REFERENCES}

Ahmad, N., Nawi, S., Rajasekaran, G., Maning, N., Aziz, M. N., Husin, A. and Rahman, N. I. (2010). Increased vancomycin minimum inhibitory concentration among Staphylococcus aureus isolates in Malaysia. Journal of Medical Microbioogyl 59 (Pt 12), 1530-1532.

Campanile, F., Mongelli, G., Bongiorno, D., Adembri, C., Ballardini, M., Falcone, M., Menichetti, F., Repetto, A., Sabia, C., Sartor, A., Scarparo, C., Tascini, C., Venditti, M., Zoppi, F. and Stefani, S. (2013). Worrisome trend of new multiple mechanisms of linezolid resistance in staphylococcal clones diffused in Italy. Journal of Clinical Microbiology 51, 1256-1259.

CLSI. (2013). Performance standards for antimicrobial susceptibility testing; Twenty-third informational supplement. CLSI document M100-S23.

Ghaznavi-Rad, E., Shamsudin, M. N., Sekawi, Z., Khoon, L. Y., Aziz, M. N., Hamat, R. A., Othman, N., Chong, P.P., van Belkum, A., GhasemzadehMoghaddam, H. and Neela, V. (2010). Predominance and emergence of clones of hospital-acquired methicillin-resistant Staphylococcus aureus in Malaysia. Journal of Clinical Microbiology 48, 867-872.

Ikeda-Dantsuji, Y., Hanaki, H., Nakae, T., Takesue, Y., Tomono, K., Honda, J., Yanagihara, K., Mikamo, H., Fukuchi, K., Kaku, M., Kohno, S. and Niki, Y. (2011). Emergence of linezolid-resistant mutants in a 
susceptible-cell population of methicillin-resistant Staphylococcus aureus. Antimicrobial Agents and Chemotherapy 55, 2466-2468.

Kos, V. N., Desjardins, C. A., Griggs, A., Cerqueira, G., Van Tonder, A., Holden, M. T., Godfrey, P., Palmer, K. L., Bodi, K., Mongodin, E. F., Wortman, J., Feldgarden, M., Lawley, T., Gill, S. R., Haas, B. J., Birren, B. and Gilmore, M. S. (2012). Comparative genomics of vancomycin-resistant Staphylococcus aureus strains and their positions within the clade most commonly associated with methicillin-resistant $S$. aureus hospital-acquired infection in the United States. MBio 3, 00112-00112.

Leach, K. L., Swaney, S. M., Colca, J. R., McDonald, W. G., Blinn, J. R., Thomasco, L. M., Gadwood, R. C., Shinabarger, D., Xiong, L. and Mankin, A. S. (2007). The site of action of oxazolidinone antibiotics in living bacteria and in human mitochondria. Molecular Cell 26, 393-402.

Liu, C., Bayer, A., Cosgrove, S. E., Daum, R. S., Fridkin, S. K., Gorwitz, R. J., Kaplan, S. L., Karchmer, A. W., Levine, D. P., Murray, B. E., Rybak, M. J., Talan, D. A. and Chambers, H. F. (2011). Clinical practice guidelines by the infectious diseases society of america for the treatment of methicillin-resistant Staphylococcus aureus infections in adults and children: executive summary. Clinical Infectious Diseases 52, 285-292.

Lodise, T. P., Lomaestro, B., Graves, J. and Drusano, G. L. (2008). Larger vancomycin doses (at least four grams per day) are associated with an increased incidence of nephrotoxicity. Antimicrobial Agents and Chemotherapy 52, 1330-1336.

Magiorakos, A. P., Srinivasan, A., Carey, R. B., Carmeli, Y., Falagas, M. E., Giske, C. G., Harbarth, S., Hindler, J. F., Kahlmeter, G., Olsson-Liljequist, B., Paterson, D. L., Rice, L. B., Stelling, J., Struelens, M. J., Vatopoulos, A., Weber, J. T. and Monnet, D. L. (2012). Multidrug-resistant, extensively drug-resistant and pandrug-resistant bacteria: An international expert proposal for interim standard definitions for acquired resistance. Clinical Microbiology and Infection 18, 268-281.

Rybak, M. J., Lomaestro, B. M., Rotschafer, J. C., Moellering, R. C., Jr. Craig, W. A., Billeter, M., Dalovisio, J. R. and Levine, D. P. (2009). Therapeutic monitoring of vancomycin in adults summary of consensus recommendations from the American Society of Health-System Pharmacists, the Infectious Diseases Society of America, and the Society of Infectious Diseases Pharmacists. Pharmacotherapy 29, 1275-1279.

Schlunzen, F., Zarivach, R., Harms, J., Bashan, A., Tocilj, A., Albrecht, R., Yonath, A. and Franceschi, F. (2001). Structural basis for the interaction of antibiotics with the peptidyl transferase centre in eubacteria. Nature 413, 814-821. 